\title{
Update on body composition tools in clinical settings: computed tomography, ultrasound, and bioimpedance applications for assessment and monitoring
}

\author{
Kathleen L. Price ${ }^{1} \cdot$ Carrie P. Earthman ${ }^{1}$ \\ Received: 29 August 2018 / Revised: 11 October 2018 / Accepted: 13 October 2018 / Published online: 30 October 2018 \\ (c) Springer Nature Limited 2018
}

\begin{abstract}
Patients with acute/critical illness are particularly vulnerable to muscle loss and fluid shifts, which adversely impact clinical outcomes. Assessment of these parameters in hospital settings is often subjective and imprecise, which creates discrepancies in identification and difficulty in assessing longitudinal changes. Body composition (BC) technologies provide objective information about muscle and fluid status that can enhance clinical assessment, and $\mathrm{BC}$ variables could be biomarkers for prognosis and targets to monitor intervention. There is growing interest in computed tomography (CT), ultrasound (US), and bioimpedance techniques as bedside assessment tools in clinical populations, and specific muscle measures, whole-body BC estimates, and select BC variables show promise as biomarkers of muscle health, nutrition risk, and fluid status. This brief review highlights work within the past 5 years on the use of BC variables generated from CT, US, and bioimpedance in clinical populations with an emphasis on those with acute/critical illness and a brief discussion of implementation challenges in these populations. Consensus on measurement protocols will facilitate identification of BC targets that best reflect prognosis and outcomes and will ultimately allow clinicians to identify individuals who would benefit most from targeted nutrition and physical therapy interventions and reliably monitor their response to treatment.
\end{abstract}

\section{Introduction}

Clinicians around the world recognize that sarcopenia, or the loss of muscle mass and/or function, and fluid overload adversely impact clinical outcomes and prognosis; thus, there is growing interest in body composition (BC) assessment techniques that can be applied in the hospital setting. The primary methods that have been explored include computed tomography (CT), ultrasound (US), and bioimpedance; comprehensive reviews of these techniques are available elsewhere [1-5]. Recent investigations using these techniques have focused on evaluating muscle quantity and quality; identifying $\mathrm{BC}$ variables that reflect wholebody lean tissue as a key component of nutritional status; and diagnosing and managing fluid overload. This brief review will highlight work within the past 5 years focusing

Carrie P. Earthman

cearthma@umn.edu

1 Department of Food Science and Nutrition, University of Minnesota-Twin Cities, St Paul, MN, USA on how BC variables generated from CT, US, and bioimpedance can be used for both their prognostic value and as biomarkers for fluid and/or nutrition status in clinical populations with an emphasis on acute/critical illness.

\section{CT}

Because $\mathrm{CT}$ imaging exposes patients to radiation, research on this BC method is largely limited to retrospective analysis for those who require the imaging in accordance with routine care, such as individuals with cancer and critical illness/trauma [3]. Quantitative measures of skeletal muscle (SM) from the third lumbar (L3) vertebra, which includes the left and right psoas, as well as paraspinal and abdominal muscles, have been examined for their prognostic value [1]. Abdominal SM cross-sectional area (CSA) from a singleslice image at L3 correlated well with whole-body SM estimates by Dual-energy X-Ray absorptiometry (DXA) in cancer [6], but it is unknown how well this relationship holds in acute/critical illness. Furthermore, CSA has been indexed to squared height to determine SM index (SMI), which correlates to increased morbidity/mortality in various 
cancer populations, and gender-specific L3 SMI sarcopenia cutpoints in cancer have been well established [1]. CTderived L3 cutpoints have been proposed to define sarcopenia in other populations including critical illness [7] and liver failure [8]. Recent CT research has three foci: (1) assessment of muscle quality by Hounsfield unit average calculation (HUAC) for the muscle tissue within the image; (2) investigation of muscle measures in acute/critical illness; and (3) evaluation of muscle measures in anatomical locations beyond the $\mathrm{L} 3$.

Current work in critical illness mimics findings in cancer, in that $\mathrm{CT}$ muscle measures predict outcomes and may be important in guiding interventions. A recent investigation in mechanically ventilated critically ill patients demonstrated that lower L3 SMI correlated with increased mortality [7]. In trauma patients, assessment of psoas HUAC better reflected poor muscularity than psoas muscle index (PMI), and HUAC was more predictive of morbidity/mortality [9]. These findings have been replicated in cancer, causing many to view HUAC measures as superior to those of quantity for predicting prognosis [1]. It has also been demonstrated that the subjective global assessment used to classify nutrition status does not adequately agree with classifications of sarcopenia based on L3 SMI for intensive care unit (ICU) patients with respiratory failure, especially for those who were male, minorities, and/or obese [10]; these data suggest that $\mathrm{CT}$ muscle analysis may improve nutritional risk classification. CT-measured loss of muscle CSA also correlated with lowered energy and protein intake in this population, suggesting that this indicator may be responsive to nutrition intervention [11].

CT-derived muscle measures may also be useful for those requiring invasive surgical intervention. Lower PMI predicted increased morbidity/mortality after liver transplant [12], and HUAC was superior to SMI in predicting waitlist mortality, though both PMI and HUAC only modestly predicted muscle function [13]. Other investigators noted that psoas muscle HUAC predicted morbidity/mortality in enterocutaneous fistula repair [14]. Preoperative pectoralis HUAC better predicted mortality than other traditional risk assessments after left ventricular assist device placement in heart failure [15]. Ultimately, these data indicate that available preoperative CT images may provide invaluable data on muscularity that can predict outcomes and determine appropriateness for invasive treatment in a number of populations.

Finally, with the freely available ImageJ software from the National Institutes of Health, increasing numbers of clinical researchers are including CT imaging in studies that explore nutrition and/or physical therapy interventions targeting muscle. Although ImageJ requires manual tracing of the muscle, a recent tutorial [16] has made the software an easily accessible tool for CT-derived $\mathrm{BC}$ analysis that provides comparable results to SliceOmatic when appropriately utilized [17]. Future directions for CT-derived BC include: further identification of appropriate scan locations available from routine care; establishment of consensus regarding best target to measure (SMI or HUAC); and defining appropriate cutoff values to define sarcopenia for specific populations. Ultimately, this work may generate an objective biomarker to diagnose sarcopenia, determine appropriateness for aggressive treatment, screen for nutritional risk, and monitor the impact of nutrition intervention.

\section{Primary challenges to the use of $\mathrm{CT}$ for assessment/monitoring in acute/critical illness}

Unlike bioimpedance and US, CT is not a bedside technique and requires patient transportation to the instrument. Importantly, radiation exposure limits $\mathrm{CT}$ to only those requiring it for medical purposes; thus, its use as an assessment/monitoring tool for muscle health is inherently limited and retrospective. Furthermore, using CT imaging to assess sarcopenia from reference cutpoints or to track muscle changes longitudinally is subject to errors caused by differences in patient positioning, the use of contrast dye (the effect of which on muscle measures is not well studied), technician variability, voltage and other device differences, and physiologic factors [2]. For example, CT scanners cannot accommodate very large individuals, and water (Hounsfield Unit, $\mathrm{HU}=0$ ) cannot be distinguished from SM (HU -29 to 150 , typically), making it difficult to interpret scans when edema is present. Finally, learning how to access and analyze CT images is a major barrier for clinicians who are pressed for time. Ultimately, collaboration with radiologists who could provide muscle measures in response to physician order would greatly facilitate the use of CT as a bedside assessment/monitoring tool.

\section{US}

US imaging is a practical, non-invasive, and readily available method for the prospective assessment of SM changes in response to illness and treatment [18]. Multi-site measurement protocols have been validated to whole-body SM by both magnetic resonance imaging (MRI) and DXA in healthy individuals $[19,20]$ and there is growing interest in the clinical application of US for objective assessment of muscle health and function earlier than tests that require volition in the critically ill [21].

Most ICU studies have focused on measurements of muscle quantity (muscle thickness and CSA) in both the quadriceps, a muscle region known to be rapidly impacted 
by sarcopenia and to correlate with ICU survival [18, 22], and the diaphragm, which is impacted by mechanical ventilation [23]. US analysis of both regions has provided insight into the pathophysiology of ICU-acquired weakness and ventilator-induced diaphragmatic dysfunction. For example, US detected quadriceps muscle thickness loss within the first 10 days of ICU stay, and more severe loss was observed with multiple organ failure than in single organ failure [24]. Quadriceps muscle thickness also predicted increased morbidity/mortality in ICU patients and has been suggested to be an objective biomarker to determine fitness for aggressive treatment [7, 25]. Additionally, in patients with respiratory failure on mechanical ventilation, the greatest mean diaphragm muscle thickness loss occurred within the first $72 \mathrm{~h}$ of ventilation, and total loss increased with extended ventilation time [23]. US measures of muscle loss in the critically ill will aid in the development of appropriate intervention strategies.

Alternatively, qualitative muscle evaluation through the measure of echogenicity (using image gray-scale analysis) has been shown to be a more sensitive indicator of muscle atrophy than quantitative measures [26] and can be used to assess necrosis, fatty infiltration, and inflammation in place of invasive muscle biopsy in critically ill patients [27, 28]. Parry and colleagues were the first to demonstrate that quadriceps echogenicity predicted muscle function in ICU patients requiring mechanical ventilation [21], corroborating earlier reports whereby this measure correlated with muscle strength independent of age and muscle quantity in an elderly population [29]. Echogenicity, then, may be an important tool for early non-volitional assessment of muscle function in the critically ill.

Ultimately, both quantitative and qualitative US measures of the quadriceps and other muscles provide tools to prospectively monitor SM change in hospital populations. US characterization of muscle changes would facilitate development of muscle-targeted nutrition and physical therapy interventions.

\section{Primary challenges to the use of US for assessment/monitoring in acute/critical illness}

There is not yet international consensus on optimal protocol to obtain reliable measurements of muscle mass and quality at the bedside [2, 18]. Studies do not universally report measurement protocol details, and a number of factors can introduce errors, particularly in longitudinal measurements [18]. For example, inconsistent landmarking and variable force of compression can influence muscle quantity; maximal compression has been shown to yield less reliability than minimal compression [30] and may be less closely aligned with other BC measures. A recent multi-site study using unilateral quadriceps measurements and maximal compression yielded only modest agreement with CTdefined low muscularity [31]. Further, there is no consensus on the best measurement sites in hospitalized patients. Although well validated in healthy individuals, a nine-site protocol [19] is not feasible in acute/critical illness. A fivesite protocol utilizing both quadriceps and one bicep [30] holds promise, particularly with minimal compression; this protocol yielded excellent inter- and intrarater reliability in healthy individuals and requires validation in hospital populations. Harris-Love et al. [32] demonstrated that both the applied force and the angle or roll (anisotropy) of the probe can introduce significant errors to measures of muscle quantity and quality; these factors will need to be quantifiable for best accuracy longitudinally, and commonly used US devices do not currently have the capacity to register force and anisotropy. Finally, obesity and edema present challenges for US measurements. These physiological states can cause inconsistent landmarking and inadequate visualization of the muscle group of interest. Significant edema can cause difficulties obtaining clear delineation of muscle. Additional research is needed to determine how best to minimize errors in these circumstances. Ideally, longitudinal measurements would benefit from the ability to record the angle/roll and force of the probe in order to maintain consistency in these variables. Following the establishment of a practical, standardized protocol, US measures of muscle quantity and quality may characterize muscle atrophy and guide clinical interventions.

\section{Bioimpedance}

Bioimpedance is the most widely utilized and studied BC assessment method for bedside application. Given its basis in electrical conductivity, bioimpedance is best designed to evaluate fluid volumes, and secondarily the lean tissue compartment (fat-free mass; FFM). There are three primary approaches to the clinical application of bioimpedance: single-frequency (SF) bioelectrical impedance analysis (BIA), multi-frequency (MF) BIA, and bioimpedance spectroscopy (BIS). It is imperative to understand the differences in underlying theory and assumptions inherent in each of these approaches, and extensive discussions of these techniques is available elsewhere [4, 5]. Bioimpedance techniques are being applied in novel ways, including the evaluation of wound healing, cardiac output, and other clinical targets [33]; however, this review will focus on recent developments in the use of bioimpedance to assess nutritional and fluid status. In general, bioimpedance 
techniques are considered accurate at the mean level for estimating FFM from total body water (TBW) estimates in healthy adults when compared with appropriate reference methods. Interestingly, the recent definition of malnutrition by the European Society of Parenteral and Enteral Nutrition includes FFM index (FFMI) defined by an SF-BIA equation. However, estimation of whole-body FFM and other compartments by bioimpedance predictive equations is error prone in acute/critical illness due to likely violation of underlying assumptions, including consistent hydration of FFM at $73 \%$, stability of distribution between intracellular water (ICW) and extracellular water (ECW), and predictability of body geometry; these and other assumptions are likely even more subject to violation in critically ill patients with obesity [4, 5]. To address this discrepancy, researchers are interested in a number of bioimpedance variables, using approaches other than whole-body SF-BIA.

Estimation of ICW and ECW is theoretically possible only with MF-BIA and BIS approaches, as SF-BIA devices only measure at one frequency $(50 \mathrm{kHz})$. Currently, wholebody and segmental MF-BIA and BIS algorithms are being utilized to assess and monitor ECW and ICW volumes in fluid overload in various clinical populations. In two recent examples, ECW/TBW estimates by segmental MF-BIA measurements (InBody S10; Biospace) using device software could be used to evaluate volume overload and predict morbidity/mortality in individuals with acute kidney injury on continuous renal replacement therapy [34]. Others have utilized whole-body BIS (Body Composition Monitor, BCM; Fresenius) using device software to assess fluid and nutritional status in patients on dialysis [35, 36], track ECW changes before and after general anesthesia [37], and monitor TBW in critically ill patients on mechanical ventilation [38]. BIS-estimated volumes agreed fairly well with clinically estimated fluid balance post anesthesia [37] but not as well in critically ill patients [38]. Further evaluation and refinement of this and other MF-BIA and BIS algorithms for fluid and nutritional monitoring in acute/critical illness is warranted.

Recent work in the elderly suggests that ECW/ICW could be a biomarker of muscle health and/or nutritional status. Segmental upper leg ECW/ICW using BIS (SFB7; ImpediMed) with device software using Cole modeling and modified Xitron mixture equations has shown promise as a marker of muscle quality in older adults, with higher ECW/ ICW correlating with lower knee extension strength and gait speed [39]. Whole-body lean tissue index (LTI) generated from a BIS device algorithm (BCM, Fresenius) correlated well with muscle strength [35] and survival [36], and an algorithm-specific cut-point (LTI $<10 \%$ of normal) to define malnutrition independently predicted mortality in patients on hemodialysis [36]. Fat mass estimates by a similar model underlying this BCM approach yielded good agreement with DXA when applied to NHANES data, and more variable results in women undergoing bariatric surgery [40]. It should be noted that bioimpedance errors increase with adiposity [5], and it is unlikely that any algorithm can be relied upon for accurate whole-body estimates in acutely ill patients with obesity.

Consequently, recent clinical bioimpedance investigations have focused on the use of "raw" bioimpedance variables, including $50 \mathrm{kHz}$ phase angle (PA), $200 / 5 \mathrm{kHz}$ impedance ratio (IR) and others as markers of nutritional status and/or prognosis [41]. Low PA has been well demonstrated to independently predict sarcopenia and mortality in a range of clinical populations. In two recent examples, low PA at ICU admission measured by different SF-BIA devices independently predicted 90-day [42] and 28-day [43] mortality in critically ill patients. Similarly, PA and IR measured by a MF-BIA device (Quadscan, BodyStat LTD) predicted ICU length of stay in opposing directions in critically ill patients in a multicenter study [44]. In addition to PA, BIS variables including characteristic frequency and cell membrane capacitance also strongly predicted knee extensor strength in the elderly [45]; these BIS-generated variables merit further exploration as biomarkers of muscle health in the clinical setting. Finally, bioelectrical vector analysis (BIVA) using $50-\mathrm{kHz}$ resistance and reactance measures has successfully been utilized as a tool to evaluate fluid management in critically ill patients [46]. Overhydration determined by BIVA has been shown to predict mortality in heart failure [47], acute kidney injury [48], and other critical illness [49].

While there is evidence to suggest that several different bioimpedance variables relate to prognosis, muscle function, and nutritional status, it is not yet clear if they can be tracked over time to reflect clinically meaningful changes in muscle health or nutritional status. Further research is warranted to determine whether select whole-body estimates by MF-BIA and BIS (e.g., ECW/ICW or LTI) and/or raw bioimpedance parameters (e.g., $50 \mathrm{kHz} \mathrm{PA}, 200 / 5 \mathrm{kHz}$ or some other high-to-low-frequency IR, characteristic frequency, or membrane capacitance) can be used as biomarkers to assess and monitor nutritional status and response to muscle-targeted interventions in acute/critical illness.

\section{Primary challenges to the use of bioimpedance for assessment/monitoring in acute/critical illness}

Different bioimpedance approaches vary widely, and device software equations and algorithms are often unknown; these 
differences are clinically problematic. As SF-BIA and MFBIA prediction equations are population specific, BIS algorithms have different resistivity constants that should be considered [50]. Clinicians often lack sufficient time to consider which algorithm is being used in the device at hand, creating confusion on how best to use the device output. Regardless of absolute accuracy, evidence suggests utility in MF-BIA and BIS whole-body ECW/ICW measures as a reflection of fluid status, and even lean estimates may be biomarkers of prognosis and/or nutritional status. Conversely, PA, IR, and other raw variables circumvent many difficulties in whole-body extrapolation; however, device, population, gender, and body mass index specificity in reference cutpoints in these parameters will impact interpretation. There are many factors that can introduce error to and influence interpretation of bioimpedance measurements in the hospital setting $[4,5]$. The most important of these include: inability to adequately position the patient for supine measures (semi-recumbent positioning is standard in ICU) with complete separation of legs, and arms from the trunk (particularly in obesity); elevated skin temperature with fever; substantial fluid and electrolyte shifts; poor skin integrity interfering with electrode placement; and close proximity of other medical devices that may be difficult to sufficiently distance from the bioimpedance device. Additionally, ICU patients have likely been lying supine for hours prior to measurements causing fluid redistribution towards the trunk; this can make it challenging to interpret results in light of reference cutpoints, which are typically generated from 10-min post-supine measurements [4]. Consistency in device and measurement protocol for longitudinal measurements will likely mitigate some of these challenges.

\section{Conclusion}

CT, US, and bioimpedance approaches provide promising clinical biomarkers for prognosis, nutritional status, and muscle health, and their use is expanding in ICU settings. Their utility in tracking response to muscle- and nutritiontargeted interventions in acute/critical illness requires additional research, which is ongoing in many major medical centers around the world. Ultimately, data from these devices would reduce subjectivity in assessment of muscularity by nutrition professionals, which could provide more sensitive screening for those at nutritional risk, as well as improve monitoring and evaluation of targeted nutrition intervention in hospital patients. Following the establishment of standardized protocols for measurement and interpretation, their utilization could particularly improve the standard of care in the ICU, where loss of muscle severely impacts prognosis and quality of life.

\section{Compliance with ethical standards}

Conflict of interest KLP declares that she has no conflict of interest. CPE has accepted loaner bioimpedance devices and/or small unrestricted funding from ImpediMed, InBody USA, and Bodystat LTD.

\section{References}

1. Paris M, Mourtzakis M. Assessment of skeletal muscle mass in critically ill patients: considerations for the utility of computed tomography imaging and ultrasonography. Curr Opin Clin Nutr Metab Care. 2016;19:125-30.

2. Teigen LM, Kuchnia AJ, Mourtzakis M, Earthman CP. The use of technology for estimating body composition: strengths and weaknesses of common modalities in a clinical setting. Nutr Clin Pract. 2017;32:20-9.

3. Prado CMM, Heymsfield SB. Lean tissue imaging: a new era for nutritional assessment and intervention. J Parent Enter Nutr. 2014;38:940-53.

4. Earthman CP. Body composition tools for assessment of adult malnutrition at the bedside: a tutorial on research considerations and clinical applications. J Parenter Enter Nutr. 2015;39:787-822.

5. Mulasi U, Kuchnia AJ, Cole AJ, Earthman CP. Bioimpedance at the bedside: current applications, limitations, and opportunities. Nutr Clin Pract. 2015;30:180-93.

6. Mourtzakis M, Prado CMMM, Lieffers JR, Reiman T, McCargar LJ, Baracos VE. A practical and precise approach to quantification of body composition in cancer patients using computed tomography images acquired during routine care. Appl Physiol Nutr Metab. 2008;33:997-1006. http://www.ncbi.nlm.nih.gov/pubmed/ 18923576

7. Weijs PJM, Looijaard WGPM, Dekker IM, Stapel SN, Girbes AR, Oudemans-vanStraaten HM, et al. Low skeletal muscle area is a risk factor for mortality in mechanically ventilated critically ill patients. Crit Care. 2014;18:R12. http://www.pubmedcentral. nih.gov/articlerender.fcgi?artid $=4028783 \&$ tool $=$ pmcentrez\& rendertype $=$ abstract.

8. Carey EJ, Lai JC, Wang CW, Dasarathy S, Lobach I, MontanoLoza AJ, et al. A multicenter study to define sarcopenia in patients with end-stage liver disease. Liver Transplant. 2017;23:625-33.

9. Yoo T, Lo WD, Evans DC. Computed tomography measured psoas density predicts outcomes in trauma. Surg (U S). 2017;162:377-84.

10. Sheean PM, Peterson SJ, Gomez Perez S, Troy KL, Patel A, Sclamberg JS, et al. The prevalence of sarcopenia in patients with respiratory failure classified as normally nourished using computed tomography and subjective global assessment. JPEN J Parenter Enter Nutr. 2014;38:873-9. http://eutils.ncbi.nlm.nih. gov/entrez/eutils/elink.fcgi?dbfrom $=$ pubmed $\&$ id $=23980135 \&$ retmode $=$ ref\&cmd $=$ prlinks\%5Cnpapers $2: / /$ publication $/$.

11. Braunschweig Ca, Sheean PM, Peterson SJ, Gomez Perez S, Freels S, Troy KL, et al. Exploitation of diagnostic computed tomography scans to assess the impact of nutrition support on body composition changes in respiratory failure patients. JPEN J Parenter Enter Nutr. 2014;38:880-5.

12. Izumi T, Watanabe J, Tohyama T, Takada Y. Impact of psoas muscle index on short-term outcome after living donor liver transplantation. Turk J Gastroenterol. 2016;27:382-8.

13. Wang CW, Feng S, Covinsky KE, Hayssen H, Zhou LQ, Yeh $\mathrm{BM}$, et al. A comparison of muscle function, mass, and quality in liver transplant candidates: results from the functional assessment in liver transplantation study. Transplantation. 2016;100:1692-8. 
14. Lo WD, Evans DC, Yoo T. CT measured psoas density predicts outcomes after enterocutaneous fistula repair. JPEN J Parenter Enter Nutr. 2018;42:176-85.

15. Teigen LM, John R, Kuchnia AJ, Nagel EM, Earthman CP, Kealhofer $\mathrm{J}$, et al. Preoperative pectoralis muscle quantity and attenuation by computed tomography are novel and powerful predictors of mortality after left ventricular assist device implantation. Circ Hear Fail. 2017;10:e004069.

16. Gomez-Perez SL, Haus JM, Sheean P, Patel B, Mar W, Chaudhry $\mathrm{V}$, et al. Measuring abdominal circumference and skeletal muscle from a single cross-sectional computed tomography image: a stepby-step guide for clinicians using National Institutes of Health ImageJ. J Parenter Enter Nutr. 2016;40:308-18.

17. Teigen LM, Kuchnia AJ, Nagel E, Deuth C, Vock DM, Mulasi U, et al. Impact of software selection and ImageJ tutorial corrigendum on skeletal muscle measures at the third lumbar vertebra on computed tomography scans in clinical populations. J Parenter Enter Nutr. 2017;00:1-9.

18. Mourtzakis M, Parry S, Connolly B, Puthucheary Z. Skeletal muscle ultrasound in critical care: a tool in need of translation. Ann Am Thorac Soc. 2017;14:1495-503.

19. Abe T, Dabbs NC, Nahar VK, Ford MA, Bass MA, Loftin M. Relationship between dual-energy X-ray absorptiometry-derived appendicular lean tissue mass and total body skeletal muscle mass estimated by ultrasound. Int J Clin Med. 2013;04:283-6. http://www.scirp.org/journal/PaperDownload.aspx?10.4236/ijcm. 2013.46049.

20. Arbeille P, Kerbeci P, Capri A, Dannaud C, Trappe SW, Trappe TA. Quantification of muscle volume by echography: comparison with MRI data on subjects in long-term bed rest. Ultrasound Med Biol. 2009;35:1092-7. http://www.ncbi.nlm.nih.gov/pubmed/ 19394755

21. Parry SM, El-Ansary D, Cartwright MS, Sarwal A, Berney S, Koopman R, et al. Ultrasonography in the intensive care setting can be used to detect changes in the quality and quantity of muscle and is related to muscle strength and function. J Crit Care. 2015;30:1151.e9-1151.e14. https://doi.org/10.1016/j.jcrc.2015. 05.024

22. Ticinesi A, Meschi T, Narici MV, Lauretani F, Maggio M. Muscle ultrasound and sarcopenia in older individuals: a clinical perspective. J Am Med Dir Assoc. 2017;18:290-300. https://doi. org/10.1016/j.jamda.2016.11.013

23. Schepens T, Verbrugghe W, Dams K, Corthouts B, Parizel PM, Jorens PG. The course of diaphragm atrophy in ventilated patients assessed with ultrasound: a longitudinal cohort study. Crit Care. 2015;19:1-8

24. Puthucheary ZA, Rawal J, McPhail M, Connolly B, Ratnayake G, Chan $P$, et al. Acute skeletal muscle wasting in critical illness. JAMA. 2013;310:1591-600. http://www.ncbi.nlm.nih.gov/ pubmed/24108501

25. Moisey LL, Mourtzakis M, Cotton BA, Premji T, Heyland DK, Wade CE, et al. Skeletal muscle predicts ventilator-free days, ICU-free days, and mortality in elderly ICU patients. Crit Care. 2013;17:R206. http://www.pubmedcentral.nih.gov/articlerender. fcgi artid $=4055977 \&$ tool $=$ pmcentrez\&rendertype $=$ abstract.

26. Fukumoto Y, Ikezoe T, Yamada Y, Tsukagoshi R, Nakamura M, Takagi $\mathrm{Y}$, et al. Age-related ultrasound changes in muscle quantity and quality in women. Ultrasound Med Biol. 2015;41:3013-7.

27. Puthucheary ZA, Phadke R, Rawal J, McPhail MJW, Sidhu PS, Rowlerson A, et al. Qualitative ultrasound in acute critical illness muscle wasting. Crit Care Med. 2015;43:1603-11.

28. Grimm A, Teschner U, Porzelius C, Ludewig K, Zielske J, Witte OW, et al. Muscle ultrasound for early assessment of critical illness neuromyopathy in severe sepsis. Crit Care. 2013;17:R227.

29. Watanabe Y, Yamada Y, Fukumoto Y, Ishihara T, Yokoyama K, Yoshida $\mathrm{T}$, et al. Echo intensity obtained from ultrasonography images reflecting muscle strength in elderly men. Clin Interv Aging. 2013;8:993-8.

30. Paris MT, Lafleur B, Dubin JA, Mourtzakis M. Development of a bedside viable ultrasound protocol to quantify appendicular lean tissue mass. J Cachex- Sarcopenia Muscle. 2017;8:713-26.

31. Paris M, Mourtzakis M, Day A, Leung R, Watharkar S, Kozar R, et al. Validation of bedside ultrasound of muscle layer thickness of the quadriceps in the critically ill patient (VALIDUM study): a prospective multicenter study. J Parenter Enter Nutr. 2017;41:171-80.

32. Harris-Love MO, Monfaredi R, Ismail C, Blackman MR, Cleary K. Quantitative ultrasound: measurement considerations for the assessment of muscular dystrophy and sarcopenia. Front Aging Neurosci. 2014;6:1-4.

33. Lukaski HC. Evolution of bioimpedance: a circuitous journey from estimation of physiological function to assessment of body composition and a return to clinical research. Eur J Clin Nutr. 2013;67(Suppl 1):S2-9. http://www.ncbi.nlm.nih.gov/pubmed/ 23299867

34. Park KH, Shin J, Hwang JH, Kim SH. Utility of volume assessment using bioelectrical impedance analysis in critically ill patients receiving continuous renal replacement therapy: a prospective observational study. 2017;29:256-64.

35. Rymarz A, Bartoszewicz Z, Szamotulska K, Niemczyk S. The associations between body cell mass and nutritional and inflammatory markers in patients with chronic kidney disease and in subjects without kidney disease. J Ren Nutr. 2016;26:87-92.

36. Marcelli D, Usvyat La, Kotanko P, Bayh I, Canaud B, Etter M, et al. Body composition and survival in dialysis patients: results from an international cohort study. Clin J Am Soc Nephrol. 2015;10:1192-200. http://cjasn.asnjournals.org/cgi/

37. Ernstbrunner M, Kostner L, Kimberger O, Wabel P, Säemann M, Markstaller K, et al. Bioimpedance spectroscopy for assessment of volume status in patients before and after general anaesthesia. PLoS ONE. 2014;9:e111139.

38. Dewitte A, Carles P, Joannès-Boyau O, Fleureau C, Roze H, Combe $\mathrm{C}$, et al. Bioelectrical impedance spectroscopy to estimate fluid balance in critically ill patients. J Clin Monit Comput. 2016;30:227-33.

39. Yamada Y, Yoshida T, Yokoyama K, Watanabe Y, Miyake M, Yamagata $\mathrm{E}$, et al. The extracellular to intracellular water ratio in upper legs is negatively associated with skeletal muscle strength and gait speed in older people. J Gerontol - Ser A Biol Sci Med Sci. 2017;72:293-8.

40. Johnson A, Matthie J, Kuchnia A, Teigen L, Beckman L, Mager J, et al. Evaluation of advanced bioimpedance spectroscopy models for measuring body composition in healthy adults (NHANES 19992004) and those undergoing massive weight loss following Rouxen-Y gastric bypass surgery. BRASPEN J. 2017;32:193-202.

41. Lukaski HC, Kyle UG, Kondrup J. Assessment of adult malnutrition and prognosis with bioelectrical impedance analysis: phase angle and impedance ratio. Curr Opin Clin Nutr Metab Care. 2017;20:330-9.

42. Stapel SN, Looijaard WGPM, Dekker IM, Girbes ARJ, Weijs PJM, Oudemans-vanStraaten HM. Bioelectrical impedance analysis-derived phase angle at admission as a predictor of 90-day mortality in intensive care patients. Eur J Clin Nutr. 2018;1-7. https://doi.org/10.1038/s41430-018-0167-1

43. Thibault R, Makhlouf A-MM, Mulliez A, Gonzalez MC, Kekstas G, Kozjek NR, et al. Fat-free mass at admission predicts 28-day mortality in intensive care unit patients: the international prospective observational study Phase Angle Project. Intensive Care Med. 2016;42:1445-53. http://link.springer.com//10.1007/ s00134-016-4468-3

44. Kuchnia A, Earthman C, Teigen L, Cole A, Mourtzakis M, Paris $\mathrm{M}$, et al. Evaluation of bioelectrical impedance analysis in 
critically ill patients: results of a multicenter prospective study. J Parenter Enter Nutr. 2017;41:1131-8.

45. Yamada Y, Buehring B, Krueger D, Anderson RM, Schoeller DA, Binkley N. Electrical properties assessed by bioelectrical impedance spectroscopy as biomarkers of age-related loss of skeletal muscle quantity and quality. J Gerontol A Biol Sci Med Sci. 2017;72:1180-6.

46. Basso F, Berdin G, Virzì GM, Mason G, Piccinni P, Day S, et al. Fluid management in the intensive care unit: bioelectrical impedance vector analysis as a tool to assess hydration status and optimal fluid balance in critically ill patients. Blood Purif. 2013;36:192-9. http://www.karger.com?10.1159/000356366.

47. Nunez J, Mascarell B, Stubbe H, Ventura S, Bonanad C, Bodf V, et al. Bioelectrical impedance vector analysis and clinical outcomes in patients with acute heart failure. J Cardio Med. 2016;17:283-90.
48. Hise AC da R, Gonzalez MC. Assessment of hydration status using bioelectrical impedance vector analysis in critical patients with acute kidney injury. Clin Nutr. 2017;1-6. http://linkinghub. elsevier.com/retrieve/pii/S0261561417300663.

49. Samoni S, Vigo V, Reséndiz LIB, Villa G, De Rosa S, Nalesso F, et al. Impact of hyperhydration on the mortality risk in critically ill patients admitted in intensive care units: comparison between bioelectrical impedance vector analysis and cumulative fluid balance recording. Crit Care. 2016;20:1-8. https://doi.org/10.1186/ s13054-016-1269-6

50. Ward LC, Isenring E, Dyer JM, Kagawa M, Essex T. Resistivity coefficients for body composition analysis using bioimpedance spectroscopy: effects of body dominance and mixture theory algorithm. Physiol Meas. 2015;36:1529-49. https://doi.org/10. $1088 / 0967-3334 / 36 / 7 / 1529$ 